\title{
Study of active Janus particles in the presence of an engineered oil-water interface
}

\author{
Priyanka Sharan, ${ }^{\dagger, \S}$ Witold Postek, ${ }^{\ddagger}, \S$ Thomas Gemming, "Piotr Garstecki, ${ }^{\ddagger}$ and \\ Juliane Simmchen*,† \\ $\dagger$ †hysical Chemistry, TU Dresden, Zellescher Weg 19, 01069 Dresden, Germany \\ $\ddagger$ Institute of Physical Chemistry, Polish Academy of Sciences, Kasprzaka 44/52,01-224 \\ Warsaw, Poland \\ ๆInstitute of Complex Materials, Leibniz IFW Dresden, Helmholtzstrasse 20, 01069 \\ Dresden \\ $\S$ Contributed equally to this work \\ E-mail: juliane.simmchen@tu-dresden.de
}

Phone: +49351 463-37433

\section{List of Supporting Videos:}

Video S1 Motion of particles from oil-water interface to solid surface.

Video S2 Motion of particles from solid surface to oil-water interface.

Video S3 Particles moving along the pinning line of an oil dimple.

Video S4 Functioning of microfluidic 2D trap. 


\section{Details of the microfluidic setup}

\section{Fabrication of the devices}

To fabricate the devices, we first produced positive polycarbonate molds with a CNC milling machine (MSG4025, Ergwind, Poland). We then poured PDMS (Sylgard 184, Dow Corning, USA) onto the polycarbonate mold and we polymerized PDMS at $70^{\circ} \mathrm{C}$ for two hours. We then treated the polymerized negative mold with plasma generated at a corona treater (BD20, Electro-Technic Products, USA) for 30 seconds. We then immediately treated the mold in 10 mbar pressure with vapors of tridecafluoro-1,1,2,2-tetrahydrooctyl-1-trichlorosilane (United Chemical Technologies, USA) for 30 minutes. We then used the negative molds to cast PDMS positive chips. We bonded the PDMS with $0.14 \mathrm{~mm}$-thick glass slides (Brain Research Laboratories, USA) by treating them with plasma generated at a plasma cleaner (Harrick Plasma, USA), and subsequently placing them together. We rendered the surface of channels hydrophobic by filling the channels with Novec 1720 (3M, USA). When Novec 1720 evaporated from the channels at room temperature, we baked the chip at $75^{\circ} \mathrm{C}$ for one hour.

\section{The 2D trap}

The system we developed allows to exchange portions of liquids in the observation zone to start the process of motion of the active particles and to vary the concentrations of the constituents of the solution. The 2D trap provides for stable conditions for observation of the active motion of the microswimmers in a droplet and allows to remove the droplets from the observation zone for repetitive experiments in the same system.

The trap locks flat discus-shaped droplets within a microfluidic chip and is an elegant solution to the problem of analysis of particle motion on liquid-liquid interface, since the 2D trap allows for i) immobilization of an aqueous droplet shaped like a discus - such shape provides for a large surface with a small curvature; ii) metering the volumes of the droplets of 
particles and of hydrogen peroxide, and merging these droplets on-chip without the electric field; iii) instantaneous observation of particles at the liquid-liquid interface after merging of the droplets; iv) exchange of the continuous phase when a droplet is already trapped, which we used to monitor the influence of the concentration of fluorosurfactant on the movements of the particles on the liquid-liquid interface; v) facile exchange of the trapped droplets.

\section{The 2D trap workflow}

The microfluidic device that we used (Fig. S1a) consists of a centrally located circular trap and four rectangular channels branching out of the trap. Two of the four channels contained a single T-junction for generation of droplets of microparticles and droplets of hydrogen peroxide, separately. The trap contains: a space for locked droplets, shallow bypasses, and four barriers at the entrances to the four branching channels. Three of the barriers contain a slit, thus resembling a merging trap that was presented before ${ }^{1-4}$ while the fourth barrier does not contain a slit, thus resembling a metering trap that was presented before. ${ }^{1,2}$ The depth of the channels leading to the trap and the depth of the trap are $0.4 \mathrm{~mm}$. The depth of bypasses around the trap is $0.15 \mathrm{~mm}$. The width of the channels leading to the trap is $0.4 \mathrm{~mm}$. The width of the slit in the merging module is $0.16 \mathrm{~mm}$. The width of the barrier in the metering module is $0.1 \mathrm{~mm}$. The volume of the trap (without bypasses) is ca. $600 \mathrm{nl}$.

The 2D trap that we demonstrate here, can be used as a merging trap or as a metering trap. If two channels are used as outlets, the behavior of droplets flowing through the trap depends on which outlet is open, and which outlet is closed. If an outlet downstream the full barrier of the trap is open, then the trap serves as a metering trap, i.e. if a droplet larger than the trap flows through, a part of this droplet becomes locked in the trap, and the excess volume of the initial droplet continues the flow towards the outlet (Fig. S1b-c). The

circular 2D trap could be used for sequential generation of droplets with gradually changing concentration in a way similar to what we have shown before ${ }^{1-4}$ (Fig. S1d-f). If an outlet downstream the barrier with a slit is open, then the trap serves as a merging trap, so that a 
droplet smaller than the trap becomes locked in the trap, but a droplet larger than the trap passes the trap without alterations to the volume of this droplet (Fig. S1g-i). The 2D trap thus allows for an easy removal of droplets from the trap by pushing a large droplet through the barrier with a slit. Exchange of droplets in the 2D trap does not require a reversal of the flow of fluids that would be necessary to remove the droplets from the hydrodynamic traps shown to date. The circular 2D trap that we introduced here allows for a stable locking of droplets, thus enabling studies of motion of particles with limited or no interference from hydrodynamic effects. The flat part of the surface of the droplet is relatively large (more than $50 \%$ of the area of the trap, as seen in Fig. 2a in the main text), allowing to follow the motion of multiple particles during a single experiment. The trap allows for operations that were to date separately performed in metering traps and merging traps. 

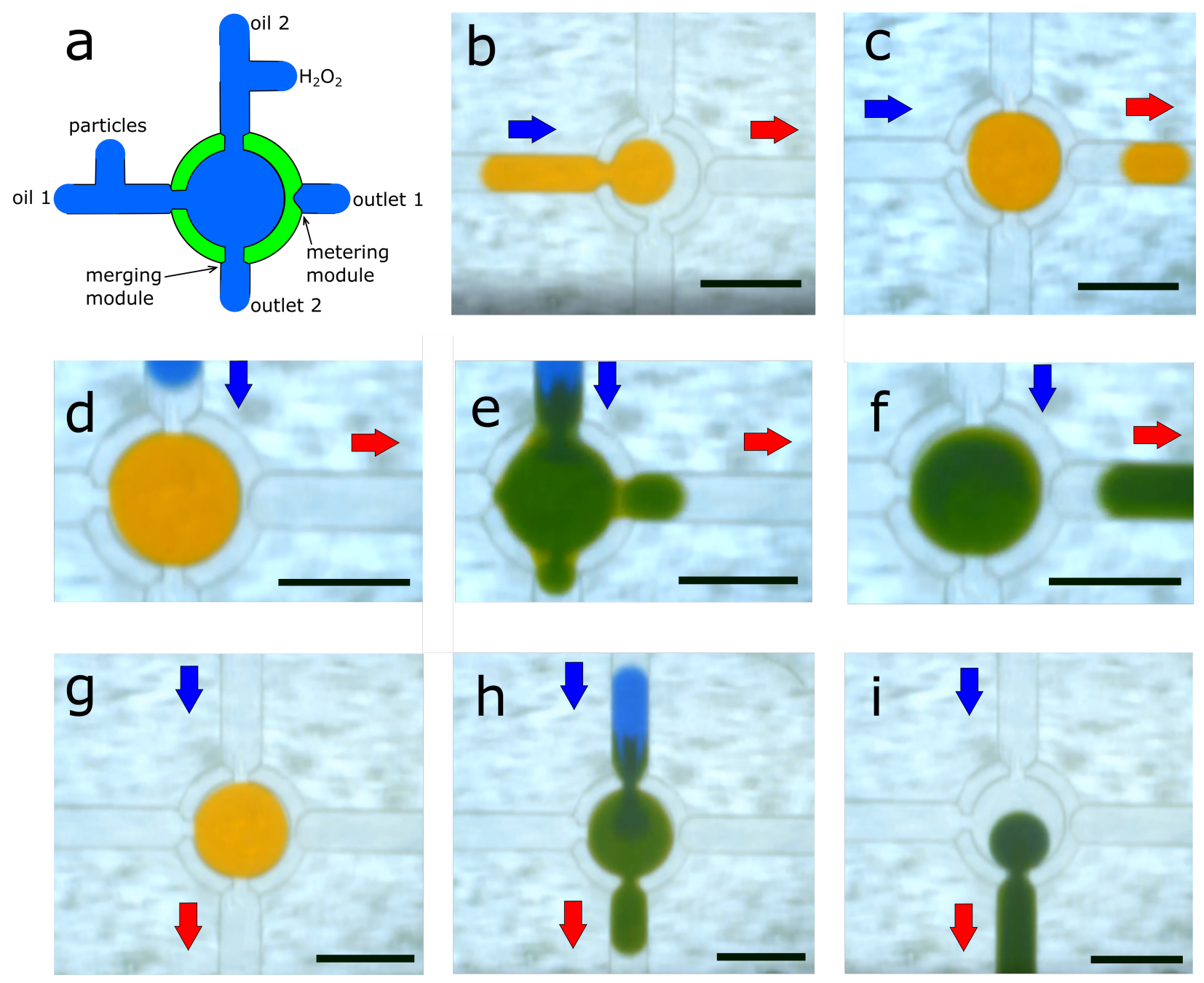

Figure S1: a) Schematics of the 2D trap. Blue: deep channels, green: shallow bypasses. The continuous phase is injected through 'oil 1' and 'oil 2' modules; b-c) Metering of volumes. A droplet of volume defined by the trap is metered and locked in the trap,; d-f) Dilution of samples; d) A droplet of sample (yellow) is locked in the trap, a droplet of diluent (blue) approaches the trap; e) Sample and of diluent mix inside the trap; f) A droplet of diluted sample is locked in the trap; g-i) Evacuating large droplets; g) A previously metered droplet is locked in the trap; h) Another droplet merges with the locked droplet and the combined droplets move over the barrier with a slit; i) A large droplet passes the trap through 'outlet 2'. Scale bars are $1 \mathrm{~mm}$. Blue arrows indicate the direction of flow from the inlets to the trap, red arrows from the trap to the outlets.

\section{Choice of fluids}

For the continuous phase, we used Novec HFE 7100 (3M, USA) with PFPE-PEG-PFPE fluorosurfactant that was synthesized according to a previously described protocol. ${ }^{5}$ We 
optimized the droplet merging conditions with respect to surfactant concentration (Figure S2). We found that at concentrations beyond $0.01 \%$, droplet merging could not be obtained. Therefore, $0.0025 \%(\mathrm{w} / \mathrm{w})$ fluorosurfactant was selected for the experiments. This concentration allowed coalescence of droplets in the traps without changing the chemical composition of the continuous phase around adjacent droplets, ${ }^{6}$ applying an electric field to the system ${ }^{2,3,7}$ or applying surface acoustic waves. ${ }^{8}$ We achieved coalescence of droplets in the $2 \mathrm{D}$ trap via compression merging. ${ }^{9}$ The dispersed phase was i) water with added microparticles, and ii) hydrogen peroxide $(5 \%(\mathrm{w} / \mathrm{w}))$. The motion of fluids was generated and controlled with single syringe infusion pumps (Cole-Parmer, USA) equipped with $1 \mathrm{~mL}$ plastic syringes.

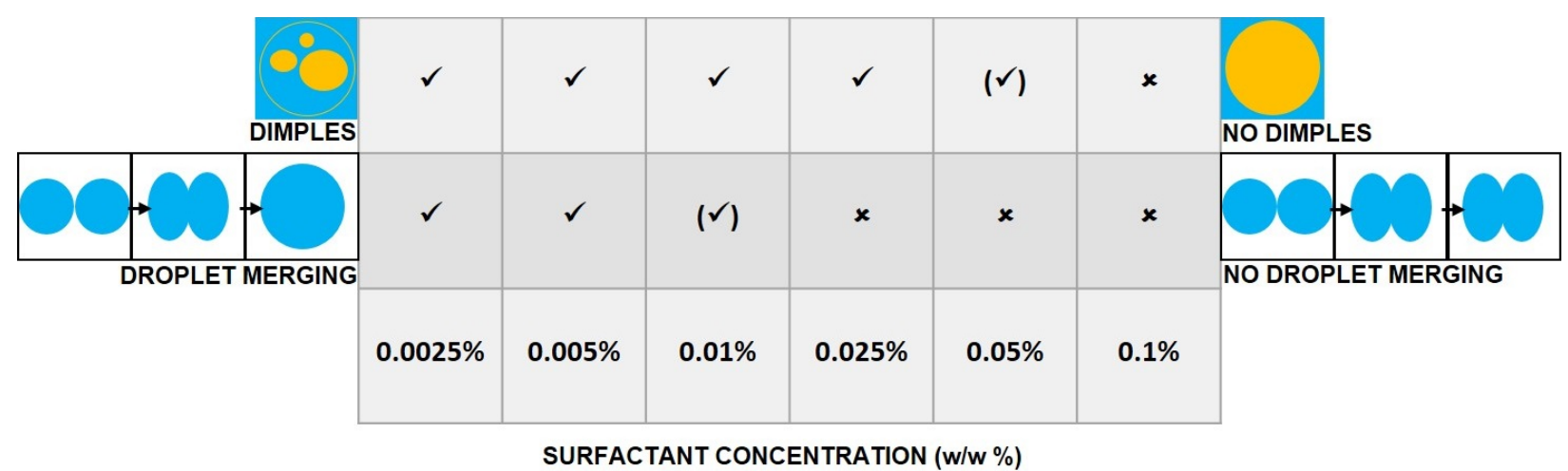

Figure S2: Optimization of droplet merging conditions with respect to change in surfactant concentration.

The large surface area of the chip resulted in a very high surface energy, which was lowered by partially wetting the substrate. This resulted in patches of oil (dimples) covering the glass substrate. The presence of these dimples was ascertained by using active $\mathrm{TiO}_{2}$ particles (Figure S3). These particles stick to the surface after activation, enabling a better visualization of oil dimples. 


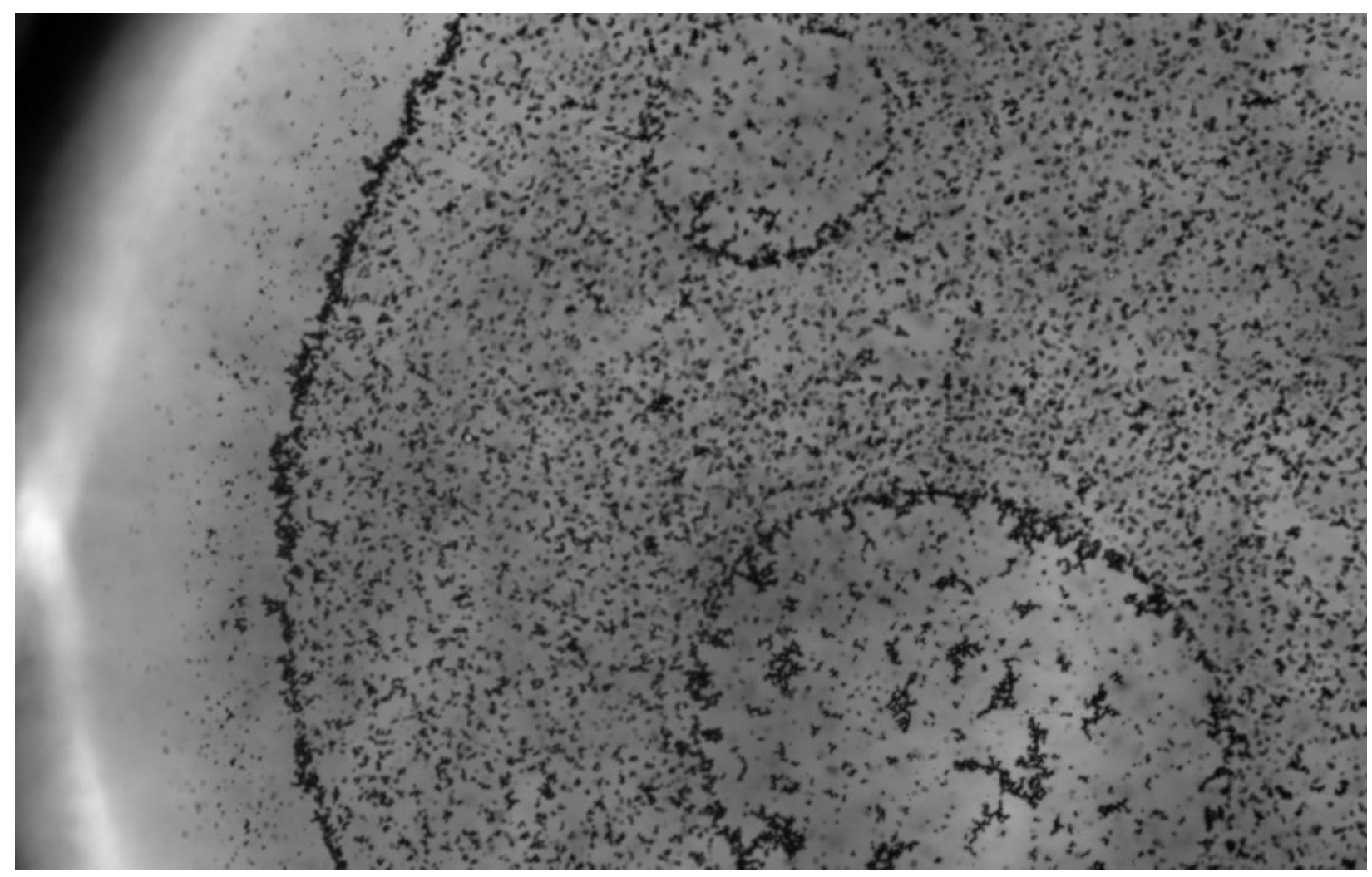

Figure $\mathrm{S} 3: \mathrm{TiO}_{2}$ particles stick to surface after activation.

\begin{tabular}{|c|c|c|c|c|c|c|c|c|}
\hline & Water & Oil & $\begin{array}{c}\text { Oil + } \\
0.0025 \% \\
\text { surfactant }\end{array}$ & $\begin{array}{c}\text { Oil }+0.005 \% \\
\text { surfactant }\end{array}$ & $\begin{array}{l}\text { Oil + } 0.01 \% \\
\text { surfactant }\end{array}$ & $\begin{array}{c}\text { Oil }+0.025 \% \\
\text { surfactant }\end{array}$ & $\begin{array}{l}\text { Oil + } 0.05 \% \\
\text { surfactant }\end{array}$ & $\begin{array}{l}\text { Oil + } 0.1 \% \\
\text { surfactant }\end{array}$ \\
\hline $\begin{array}{l}\text { Non-treated } \\
\text { glass }\left({ }^{\circ}\right)\end{array}$ & 51.1 & 12.9 & 9.3 & 11.3 & 9.3 & 10.6 & 11.4 & 11.2 \\
\hline $\begin{array}{l}\text { Hydrophobic } \\
\text { glass }\left({ }^{\circ}\right)\end{array}$ & 94.1 & 6.9 & 8.7 & 8.7 & 7.5 & 6.8 & 9.1 & 7.5 \\
\hline
\end{tabular}

Figure S4: Contact angle measurements.

\begin{tabular}{|c|c|c|c|c|c|c|c|}
\hline & Oil & $\begin{array}{c}\text { Oil +0.0025\% } \\
\text { surfactant }\end{array}$ & $\begin{array}{c}\text { Oil + } 0.005 \% \\
\text { surfactant }\end{array}$ & $\begin{array}{l}\text { Oil + } 0.01 \% \\
\text { surfactant }\end{array}$ & $\begin{array}{c}\text { Oil + } 0.025 \% \\
\text { surfactant }\end{array}$ & $\begin{array}{l}\text { Oil + } 0.05 \% \\
\text { surfactant }\end{array}$ & $\begin{array}{l}\text { Oil + } 0.1 \% \\
\text { surfactant }\end{array}$ \\
\hline $\begin{array}{l}\text { Oil in water } \\
(\mathrm{mN} / \mathrm{m})\end{array}$ & 38.4 & 36.8 & 37.4 & 34.4 & 25.1 & 24.3 & 22.2 \\
\hline $\begin{array}{l}\text { Water in Oil } \\
(\mathrm{mN} / \mathrm{m})\end{array}$ & 47.0 & 44.2 & 40.2 & 35.7 & 28.7 & 25.8 & 21.6 \\
\hline
\end{tabular}

Figure S5: Surface tension measurements of oil in water and water in oil. 


\section{Details of micromotors and imaging}

\section{Preparation of particles}

Janus particles were obtained by drop casting of a suspension of spherical silica colloids (3 $\mu \mathrm{m}$ diameter, Sigma-Aldrich) on an oxygen-plasma-cleaned glass slide followed by slow evaporation of the solvent. Subsequently, a 5 nm layer of Pt was sputtered on the monolayer. To release particles from the glass slides into de-ionized (DI) water, short ultrasound pulses were sufficient.

\section{Imaging and detection}

The formation of bubbles in the closed volume of the PDMS chip due to excessive production of oxygen at the active part of particles was avoided by using a small number of particles to keep the overall concentration of produced oxygen low. However, the gas permeability of PDMS was also beneficial since the diffusion of oxygen out of the system was enabled.

The locked droplet and the particles inside the droplet were recorded with an attached camera to a Zeiss microscope. The recordings are generated at a frame rate of 40 frames per second. The videos were analyzed with ImageJ software and the speed was obtained as an average of minimum 30 particles.

\section{References}

(1) Korczyk, P. M.; Derzsi, L.; Jakieła, S.; Garstecki, P. Microfluidic traps for hard-wired operations on droplets. Lab on a Chip 2013, 13, 4096-4102.

(2) Postek, W.; Kaminski, T. S.; Garstecki, P. A precise and accurate microfluidic droplet dilutor. The Analyst 2017, 142, 2901-2911.

(3) Postek, W.; Kaminski, T. S.; Garstecki, P. A passive microfluidic system based on 
step emulsification allows to generate libraries of nanoliter-sized droplets from microliter droplets of varying and known concentration of sample. Lab Chip 2017, 17, 1323-1331.

(4) Derzsi, L.; Kaminski, T. S.; Garstecki, P. Antibiograms in five pipetting steps: precise dilution assays in sub microliter volumes with a conventional pipette. Lab Chip 2016, 16, 893-901.

(5) Holtze, C. et al. Biocompatible surfactants for water-in-fluorocarbon emulsions. Lab on a Chip 2008, 8, 1632-1639.

(6) Akartuna, I.; Aubrecht, D. M.; Kodger, T. E.; Weitz, D. A. Chemically induced coalescence in droplet-based microfluidics. Lab Chip 2015, 15, 1140-1144.

(7) Bhattacharjee, B.; Vanapalli, S. A. Electrocoalescence based serial dilution of microfluidic droplets. Biomicrofluidics 2014, 8, 044111.

(8) Sesen, M.; Alan, T.; Neild, A. Microfluidic on-demand droplet merging using surface acoustic waves. Lab Chip 2014, 14, 3325-3333.

(9) Niu, X.; Gulati, S.; Edel, J. B.; DeMello, A. J. Pillar-induced droplet merging in microfluidic circuits. Lab on a chip 2008, 8, 1837-1841. 